\title{
EFFECTS OF VOLUNTARY HANDHELD VS. SPEECH-BASED TEXT ENTRY ON DRIVING PERFORMANCE IN (UN)PREDICTABLE CRITICAL SITUATIONS
}

\author{
Katja Schleinitz*, Tibor Petzoldt ${ }^{\#}$ \\ *TU Chemnitz, Germany / TÜV | DEKRA arge tp 21, ${ }^{\text {T TU Dresden, Germany }}$ \\ Email: katja.schleinitz@argetp21.de
}

\begin{abstract}
Summary: It is often suggested that speech-based entry systems for text messages might provide a solution for the safety problems that arise because of handheld texting. However, although such systems have the advantage of allowing drivers to keep their eyes on the road, there is still a considerable portion of cognitive load associated with texting, which might impair the processing of relevant information. At the same time, drivers do not tend to text always and everywhere, but rather only in situations, they consider "suitable". The selection of relevant test scenarios, and the free choice to (not) text in these scenarios are key aspects in the investigation of the effects of texting that have often been neglected. The aim of this experiment was to investigate the consequences of voluntary visual-manual and speech-based text messaging on reaction time and crashes in critical situations that might or might not be anticipated with the help of an environmental cue. We conducted a driving simulator study in which at one point, a child crossed the road, sometimes preceded by a ball rolling across, sometimes not. Participants (82, three groups: handheld writing, speech-based entry, control group) were free to (not) engage in a texting task while driving. While the pre-information had a positive impact on brake reaction time, there were no significant differences between the different groups in either crash rate or brake reaction time. The results highlight the role the design of test scenarios plays for the effects of texting on driving performance.
\end{abstract}

\section{INTRODUCTION}

The fact that texting while driving has the potential to negatively affect driving performance has been shown repeatedly in the lab and on the road (Caird, Johnston, Willness, Asbridge, \& Steel, 2014; Simmons, Hicks, \& Caird, 2016). Speech-based entry systems for text messages have been suggested as one potential approach to address the safety problems created by such forms of visual-manual distraction. Obviously, these systems can potentially reduce the hands-off-steeringwheel phases as well as the glances away from the road, which correlate with a higher crash risk (Klauer et al., 2006). But, at the same time, the cognitive engagement in the task still has the potential to impact on the interpretation of information that might be vital for the anticipation of a critical situation. Unfortunately, studies in that regard are rare and have produced somewhat mixed results. He et al. (2014) found that the brake response time for speech-based entry and a drive-only condition were nearly similar, but faster compared to a handheld condition. In contrast, other studies found longer reaction times for speech-based entry compared to the drive-only condition (He, Choi, McCarley, Chaparro, \& Wang, 2015; Yager, 2013).

Indeed, it is not fully clear if speech-based interaction is superior to the visual-manual entry of text messages. Muhrer and Vollrath (2011) found that while visual distraction has the potential to impair the perception of and reaction to unexpected events, cognitive distraction can impact on 
driver performance by impairing a driver's ability to predict future developments of the traffic situation. Baumann, Petzoldt, Groenewoud, Hogema, and Krems (2008) found that while engagement in a cognitive task (with no visual component) was largely irrelevant for the response in a sudden, unpredictable situation, it played an important role if the same situation was preceded by a potentially helpful environmental cue (in addition to an - unsurprising - main effect of predictability in general). Attentive drivers were able to process the information represented by the cue, adapted their behavior prior to the actual critical situation, and also responded faster once the situation occurred, whereas secondary task engagement had detrimental effects on both of these measures. It seems that even if drivers' eyes are on the road, their mind is not, resulting in relevant information being overlooked or not processed properly.

It should be noted, though, that most drivers do not seem to just text anywhere anytime. Instead, they appear to adapt their texting behavior dependent on the driving context to mitigate the potentially negative effects on driving performance, i.e, they, on average, decrease secondary task engagement in potentially critical situations, which might indicate that they are able to anticipate task demand and adapt their behavior accordingly (Petzoldt \& Schleinitz, 2018, Schömig, Schoch, Neukum, Schumacher, \& Wandtner, 2015). In fact, it might be argued that quite a few simulator studies on texting (and secondary task engagement in general) have produced inflated estimates of crash risk increase, as they required drivers to text in situations which they themselves might not find "suitable" for texting (Drews, Yazdani, Godfrey, Cooper, \& Strayer, 2009; Yannis, Laiou, Papantoniou, \& Gkartzonikas, 2016). However, while undisputedly some form of behavioral adaptation takes place, it is unclear to what extent the selection of contexts "suitable" for texting is actually appropriate. More specifically, it raises the question of how drivers deal with potentially critical situations that develop in apparently simple and safe driving contexts.

The overall aim of the experiment presented in this paper was to investigate the consequences of visual-manual and speech-based entry of text messages on drivers' performance (e.g., reaction time, crashes) in critical situations that are or are not preceded by environmental cues that would allow for an anticipation of the event. Crucially, the situations in which these events occurred were supposed to be designed that they would be considered "suitable" for texting, and drivers were supposed to be free to (not) engage in the secondary task. This resulted in an overall experimental design which included three different secondary task conditions ("handheld writing", "speech-based entry", "control") and two predictability conditions ("with pre-information", "without pre-information").

\section{METHOD}

The reported methods (and findings) are part of a larger simulator experiment (Schleinitz, Petzoldt, Krems, Buchholz, \& Gehlert, 2018), in which not only text message entry (manual and speech based), but also text message reception (visual and auditory) were tested, and further analyses (e.g. continuous driving performance measures, such as speed, lateral position) were conducted. For the research question addressed in this paper, we focus on the behavior in a critical situation.

\section{Participants}

The dataset for the analysis presented in this paper included the drives of 82 participants ( 41 female, 41 male) with a mean age of 30.3 years (18 to 63 years). On average, they had their driving 
license for 12.5 years ( 1 to 44 years), and produced a mean annual mileage of $29,773 \mathrm{~km}(2,500$ $-350,000 \mathrm{~km})$. All were frequent texters. Nearly three quarters reported to text at least once per one-hour drive. In the reported experiment, 17 of them were assigned to the "control" group, i.e. they drove without additional secondary task, and experienced the same critical situation both with and without pre-information. The remaining participants were allocated to two "message entry" groups, one of which completed the task through "handheld writing" ( $\mathrm{n}=33)$, the other by "speech-based entry" $(n=32)$. One half of each of the two groups experienced a critical situation with pre-information, the other half without pre-information.

\section{Material}

Driving simulator. We used a static driving simulator with a fully equipped interior and a $180^{\circ}$ horizontal field of view, including two side mirrors and a rear mirror. The simulator vehicle had an automatic transmission. We created the driving scenarios using the simulation software SILAB 5.0, which also allowed for the collection of a variety of driving parameters. For a better description of the driving behavior and secondary task involvement, the vehicle was equipped with multiple cameras that recorded, e.g., the face of the driver, his/her upper body, or the interior of the car (from the back seat), so that any handling of the mobile phone was clearly visible.

Driving scenarios. An $11.7 \mathrm{~km}$ long route in urban areas was created, which consisted of ten driving scenarios with sections with $30 \mathrm{~km} / \mathrm{h}$ and $50 \mathrm{~km} / \mathrm{h}$ speed limit. These scenarios were chosen based on the results of a previous study on the influence of situational factors on texting (Schleinitz et al, 2018). In general, the route was of low demand, with little surrounding traffic, only slight curves, few pedestrians on the pavements, interspersed from time to time with intersections and parked vehicles. A trip lasted about 15 minutes, and contained a critical situation in which a child suddenly crossed the street (see Figure 1), while the participant drove on a twolane road (speed limit $50 \mathrm{~km} / \mathrm{h}$ ) with parked vehicles on the right-hand side of the road. In one version of the situation, a blue ball rolled across the road (ego vehicle $70 \mathrm{~m}$ away) before the child stepped out behind the parked vehicle (once the ball had crossed the road, i.e. $1.4 \mathrm{~s}$ after it became was visible; situation with pre-information), in another version, there was no such ball (situation without pre-information). Drivers had to slow down / brake (but not necessarily stop) to avoid a collision.
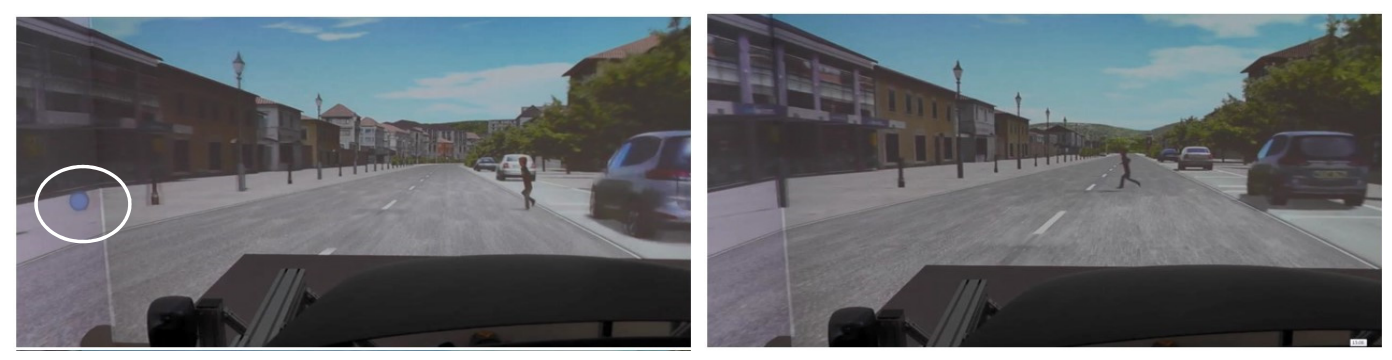

Figure 1: Driving scenario with critical situation. Left: with pre-information (ball has just crossed the road, child starting to cross); right: without pre-information

Secondary tasks. During the drive, the participants of the two "message entry" groups were asked to "write" ten text messages, each triggered by their current position along the route. One activity required a visual-manual operation of the smartphone, for one, the voice control of a sys- 
tem integrated in the vehicle (touch display in the center console) was used. The "handheld writing" group used their own phone for message entry, "speech-based entry" group simply had to push a button on a touch screen (center stack), wait for a signal, and then verbalize the message, before giving the voice command "send" to finish the interaction. To give the message a certain "naturalistic touch", participants were presented with a topic (e.g., "last Christmas presents", played from tape as soon as they reached a certain trigger point), and were instructed to respond with a short message of about two lines of text. There were no "correct" or "incorrect" responses. This procedure was chosen to avoid having to memorize predefined messages, and instead have participants come up with content more naturally.

\section{Procedure}

At the beginning, the participants received a brief introduction to the study, as well as information about the driving simulator, data recording and simulator sickness. Afterwards they completed a practice drive in order to get familiar with the handling of the simulated vehicle. Then the respective instructions for the trip followed. The task of the participants was to follow the traffic signs towards a certain destination. They should drive as naturally as possible, follow the traffic rules and should not be in a hurry, but not drive too slow.

In addition, the two "text entry" groups received instructions for the respective secondary task. It was explained to them that an auditory signal would indicate that they had a "texting" section on the route, i.e. it was, in principle, possible to work on the secondary task. The participants should then decide for themselves if and when the situation, in their opinion, allowed them to text, just as they would do on a regular trip. But they should not feel urged. In addition, specific instructions were given on each secondary task, e.g., touching the touch screen or how voice input works. They were informed that 30 seconds after the auditory signal had indicated the start of the "texting" section, another signal would occur indicating that the secondary task should be stopped, regardless of the actual status of the task. This was to prevent participants from being engaged in the secondary task for too long, which would have caused problems with the timing of the following tasks.

After the instructions, the participants completed two drives in the simulator (which contained the same route sections in different order to avoid learning effects). In one sequence, the child occurred with the ball as pre-information, while in the other sequence, the child crossed the street without the ball present. The order of the sequences was balanced across participants. In the original simulator study, the participants completed a different secondary task in each sequence (except for the participants of the control group). In between the two drives, participants completed a short questionnaire. The purpose of this interruption was to get the participants to leave the driving simulator and actually take a short break (also to prevent simulator sickness). After the drives, they completed the second part of the questionnaire. Overall, the experiment took 90 to 120 minutes.

\section{Data preparation and analysis}

For the analysis of the drivers' reaction to the critical situation, it was necessary to specify the section of the route on which behavioral parameters should be considered as showing an actual behavioral response to the situation. Therefore, the point at which the pre-information, i.e., the 
ball, became visible, was set as the starting point of each section. The same reference point was chosen for the scenarios in which the critical situation occurred without pre-information (i.e., the point at which this pre-information would have become visible). The point at which the child crossed the street was chosen as the end point (section length of $70 \mathrm{~m}$ ). The videos of the integrated camera system were used to identify and determine the frequency of collisions with the child. The response time for braking was defined as the time between the start of the analyzed interval (i.e., point at which (pre-)information became /would have become visible) and the first measurable operation of the brake pedal. Since data was normally distributed an ANOVA was calculated.

\section{RESULTS}

In Figure 2 left, the percentage of crashes is illustrated for the different secondary tasks and preinformation conditions. On a descriptive level, the proportion of crashes during handheld writing was higher than during speech-based entry or drive-only, especially for the condition without preinformation. The speech-based entry resulted in even fewer crashes than the control group in both conditions. Also visible is a general difference in crash rates between situations with and without pre-information, with drivers performing better when the pre-information was present.

Figure 2 right shows the time until the first operation of the brake pedal. The participants pressed the brake pedal significantly earlier $(M=2.80 ; S D=0.54)$ when they were warned by the ball than without this pre-information $\left(M=3.38 ; S D=0.46 ; F(1,81)=30.15, p=.031, \eta^{2}=0.937\right)$. Although the reaction time during handheld writing was slightly slower $(M=3.26 ; S D=0.65)$ than in the other two conditions (speech-based entry $M=3.02 ; S D=0.49$; control group $M=$ 2.96; $S D=0.54$ ), we found no significant difference between the three secondary task conditions $\left(F(2,81)=2.62, p=.276, \eta^{2} \mathrm{p}=0.724\right)$. Likewise, the post-hoc tests revealed no significant differences (all $\mathrm{p}>$.059). On a descriptive level, the reaction time during handheld writing seemed to suffer (in comparison to the other two conditions) mainly when there was pre-information presented, however, again, the ANOVA showed no significant interaction between secondary task condition and pre-information $\left(F(2,81)=1.00, p=.374, \eta^{2} \mathrm{p}=0.024\right)$.
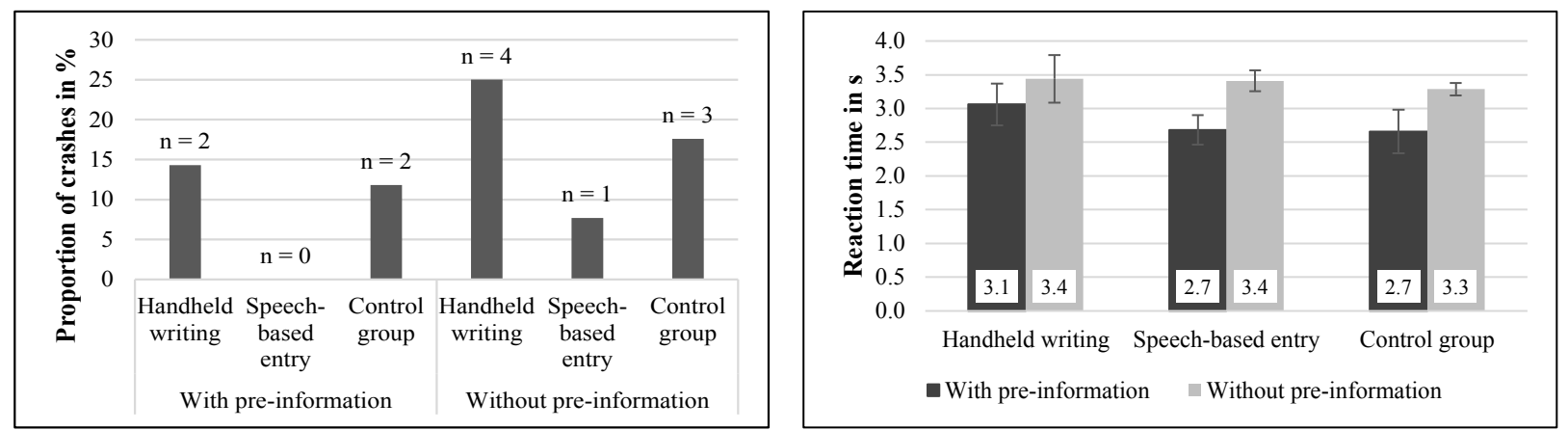

Figure 2 left: proportion of crashes for the three secondary task conditions with and without pre-information, right: mean reaction time for braking for three secondary task conditions with and without pre-information.

\section{DISCUSSION}

The aim of this driving simulator study was to investigate how the (freely chosen) engagement in 
two different forms of texting affects the reaction behavior in a critical situation with and without pre-information. As expected, the pre-information had a positive effect on the reaction time. On a descriptive level, also the number of (admittedly, rather artificial) collisions was lower. Still, it seemed that to some degree and regardless of whether participants were engaged in a secondary task or not, they were able to take advantage of the pre-information

At the same time, we found no clear-cut differences between the secondary task conditions. While on a descriptive level, the number of collisions as well as brake reaction time were higher for the handheld writing condition, the statistical analyses did not reveal any significant effects, neither in the comparison of the two "text entry" conditions, nor between them and the control condition. While the number of data points in our study (especially with regard to collisions) certainly limits the generalizability of our findings, it is interesting that brake reaction times did not differ between the two "text entry" conditions and the control condition, which is inconsistent with findings of others (Libby, Chaparro, \& He, 2013; Yannis et al., 2016) who reported performance decrements as a result of read/write a text message.

A potential explanation might be found in the characteristics of our driving scenarios, which were deliberately designed to reflect conditions which drivers would consider appropriate for texting. Indeed, all drivers in our sample chose to engage in texting in the scenario presented in this paper. And indeed, their responses when texting, both hand-held and using speech based input, were as fast (or as slow) as when they did not text, and could potentially have devoted all attention to the road ahead. This finding certainly should not be interpreted as evidence that drivers' are fully capable of selecting "appropriate" situations for texting. Overall, crash data clearly indicate that they are not. Rather, it should be seen as a slight criticism of an oversimplified or too broad interpretation of study results that report massive performance decrements when texting in highly complex driving scenarios. It should always be questioned how plausible it is that a driver would be willing to text under the investigated conditions in real traffic.

Of course, in a similar fashion, it could be questioned how representative our chosen scenarios were for potentially problematic situations on the road. Our participants' unequivocal willingness to text indicates a maximum level agreement that the depicted situation would be safe enough to engage in a secondary task. It might be argued that the presented scenarios were "too easy" for our drivers. Future studies should therefore consider implementing scenarios for which drivers' judgment is less homogenous. Still, the fact remains that ideally, investigations of the potential consequences of texting always address the combination of a driver's willingness to text in a certain situation and his / her ability to handle driving (and texting) in that situation.

\section{ACKNOWLEDGMENTS}

The research presented in this paper was funded by German Insurers Accident Research (UDV).

\section{REFERENCES}

Baumann, M., Petzoldt, T., Groenewoud, C., Hogema, J., \& Krems, J. F. (2008). The Effect of Cognitive Tasks on Predicting Events in Traffic. Proceedings of the European Conference on Human Centred Design for Intelligent Transport Systems, 3-12. 
Caird, J. K., Johnston, K. A., Willness, C. R., Asbridge, M., \& Steel, P. (2014). A meta-analysis of the effects of texting on driving. Accident Analysis and Prevention, 71, 311-318. https://doi.org/10.1016/j.aap.2014.06.005

Drews, F.A, Yazdani, H., Godfrey, C., Cooper, J. M., \& Strayer, D.L.(2009). Text messaging during simulated driving. Human Factors, 51, 762-770. doi: 10.1177/0018720809353319

He, J., Chaparro, A., Nguyen, B., Burge, R. J., Crandall, J., Chaparro, B. S., Ni, R., Cao, S. (2014). Texting while driving: Is speech-based text entry less risky than handheld text entry? Accident Analysis \& Prevention, 72, 287-295. https://doi.org/10.1016/j.aap.2014.07.014

He, J., Choi, W., McCarley, J. S., Chaparro, B. S., \& Wang, C. (2015). Texting while driving using Google Glass: Promising but not distraction-free. Accident Analysis and Prevention, 81, 218-229. https://doi.org/10.1016/j.aap.2015.03.033

Klauer, S. G., Dingus, T., Neale, V. L., Sudweek, J. D., \& Ramsey, D. J. (2006). The Impact of Driver Inattention On Near Crash/Crash Risk: An Analysis Using the 100-Car Naturalistic Driving Study Data. Blacksburg, Virginia: VTTI https://doi.org/DOT HS 810594

Libby, D., Chaparro, A., \& He, J. (2013). Distracted while driving: A comparison of the effects of texting and talking on a cell phone. In Proceedings of the Human Factors and Ergonomics Society Annual Meeting, 57, 874-1878. https://doi.org/10.1177/1541931213571418

Muhrer, E., \& Vollrath, M. (2011). The effect of visual and cognitive distraction on driver's anticipation in a simulated car following scenario. Transportation Research Part F: Traffic Psychology and Behaviour, 14(6), 555-566. https://doi.org/10.1016/j.trf.2011.06.003

Petzoldt, T. \& Schleinitz, K. (2018). To text or not to text- Drivers' interpretation of traffic situations as the basis for their decision to (not) engage in text messaging. 6th International Conference on Driver Distraction and Inattention, Gothenburg, Sweden 15.-17. October 18.

Schleinitz, K., Petzoldt, T., Krems, J., Buchholz, K., \& Gehlert, T. (2018). Fahrerablenkung durch Informations- und Kommunikationssysteme, insbesondere Textbotschaften. (Forschungsbericht Nr. 49). Berlin: Unfallforschung der Versicherer e.V.

Schömig, N., Schoch, S., Neukum, A., Schumacher, M., \& Wandtner, B. (2015). Simulatorstudien zur Ablenkungswirkung fahrfremder Tätigkeiten. (M 253). Bergisch Gladbach: BASt.

Simmons, S. M., Hicks, A., \& Caird, J. K. (2016). Safety-critical event risk associated with cell phone tasks as measured in naturalistic driving studies: A systematic review and meta-analysis. Accident Analysis and Prevention, 87, 161-169. https://doi.org/10.1016/j.aap.2015.11.015

Yager, C. E. (2013). Driver Safety Impacts of Voice-to-Text Mobile Applications. In Proceedings of the Human Factors and Ergonomics Society Annual Meeting, 57, 1869-1873. San Diego. https://doi.org/10.1177/1541931213571417

Yannis, G., Laiou, A., Papantoniou, P., \& Gkartzonikas, C. (2016). Simulation of texting impact on young drivers behavior and safety on motorways. Transportation Research Part F: Traffic Psychology and Behaviour, 41, 10-18. https://doi.org/10.1016/j.trf.2016.06.003 